\title{
Solving the Computational Puzzle: Toward a Pragmatic Pathway for Modeling Low-Energy Vibrational Modes of Pharmaceutical Crystals
}

Kārlis Bērziṇša , Joshua J. Sutton ${ }^{\mathrm{a}}$, Sara J. Fraser-Miller ${ }^{\mathrm{a}}$, Thomas Rades ${ }^{\mathrm{b}}$, Timothy M. Korter ${ }^{\mathrm{c}}$, Keith C. Gordon $^{\mathrm{a}^{*}}$

${ }^{a}$ The Dodd-Walls Centre for Photonic and Quantum Technologies, Department of Chemistry, University of Otago, Dunedin 9016, New Zealand

${ }^{b}$ Department of Pharmacy, Faculty of Health and Medical Sciences, University of Copenhagen, Copenhagen 1165, Denmark

${ }^{\mathrm{c}}$ Department of Chemistry, Syracuse University, Center for Science and Technology, Syracuse, New York 13244, United States

*Corresponding author

\section{Supporting information}
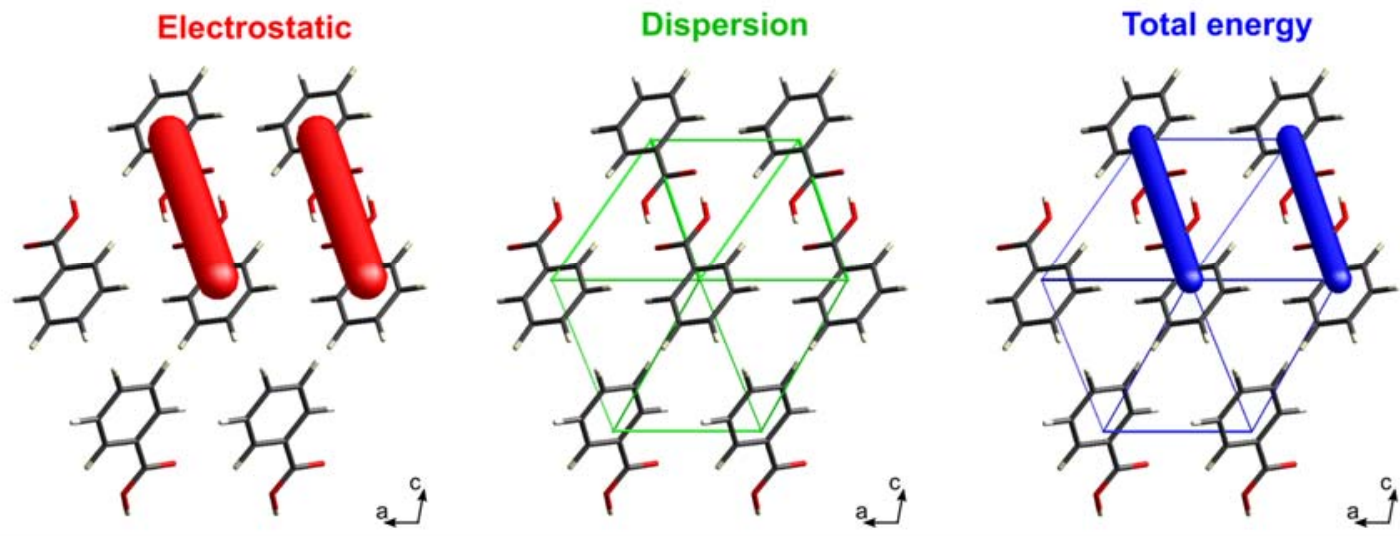

Figure S1. Energy framework diagrams for a cluster of nearest-neighbor molecules in benzoic acid. All diagrams use the same tube/cylinder scale of 50, and energies with a magnitude smaller than 5 $\mathrm{kJ} / \mathrm{mol}$ have been omitted for clarity.
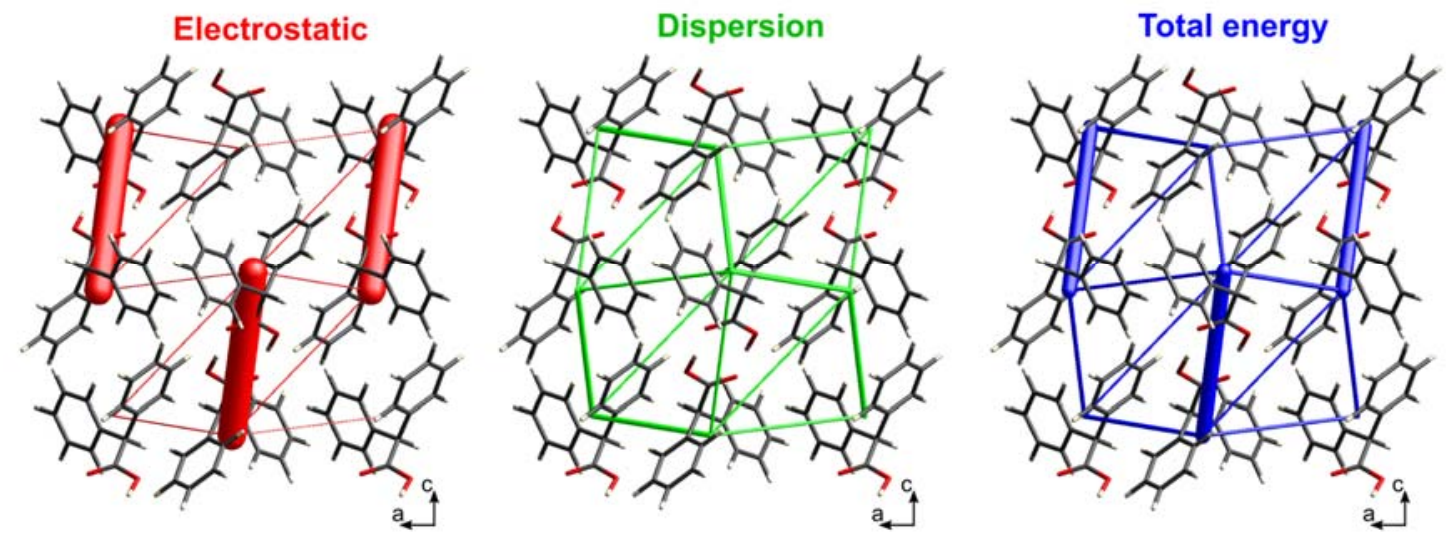

Figure S2. Energy framework diagrams for a cluster of nearest-neighbor molecules in diphenylacetic acid. All diagrams use the same tube/cylinder scale of 50, and energies with a magnitude smaller than $5 \mathrm{~kJ} / \mathrm{mol}$ have been omitted for clarity. 

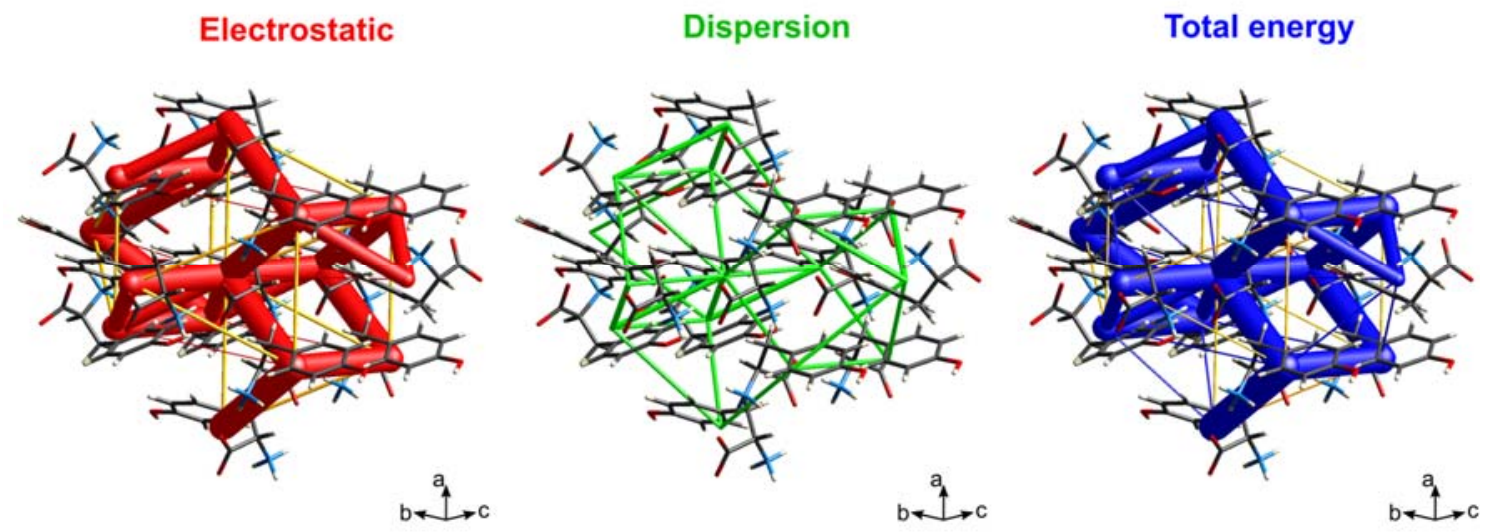

Figure S3. Energy framework diagrams for a cluster of nearest-neighbor molecules in L-tyrosine. All diagrams use the same tube/cylinder scale of 50 , and energies with a magnitude smaller than $5 \mathrm{~kJ} / \mathrm{mol}$ have been omitted for clarity. Destabilizing (positive) energies are depicted in yellow color.

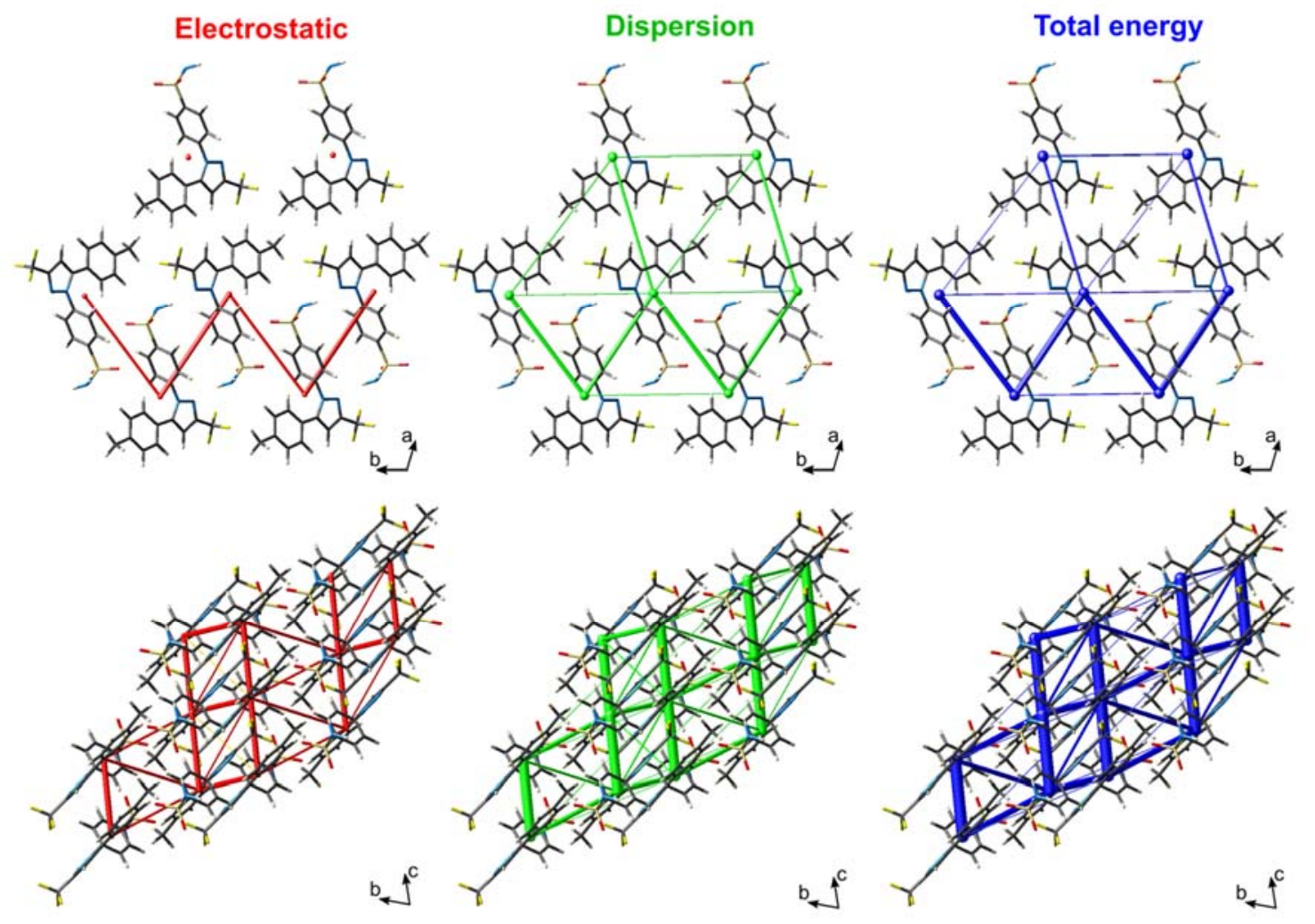

Figure S4. Energy framework diagrams for a cluster of nearest-neighbor molecules in celecoxib (form III). All diagrams use the same tube/cylinder scale of 50, and energies with a magnitude smaller than 5 $\mathrm{kJ} / \mathrm{mol}$ have been omitted for clarity. 


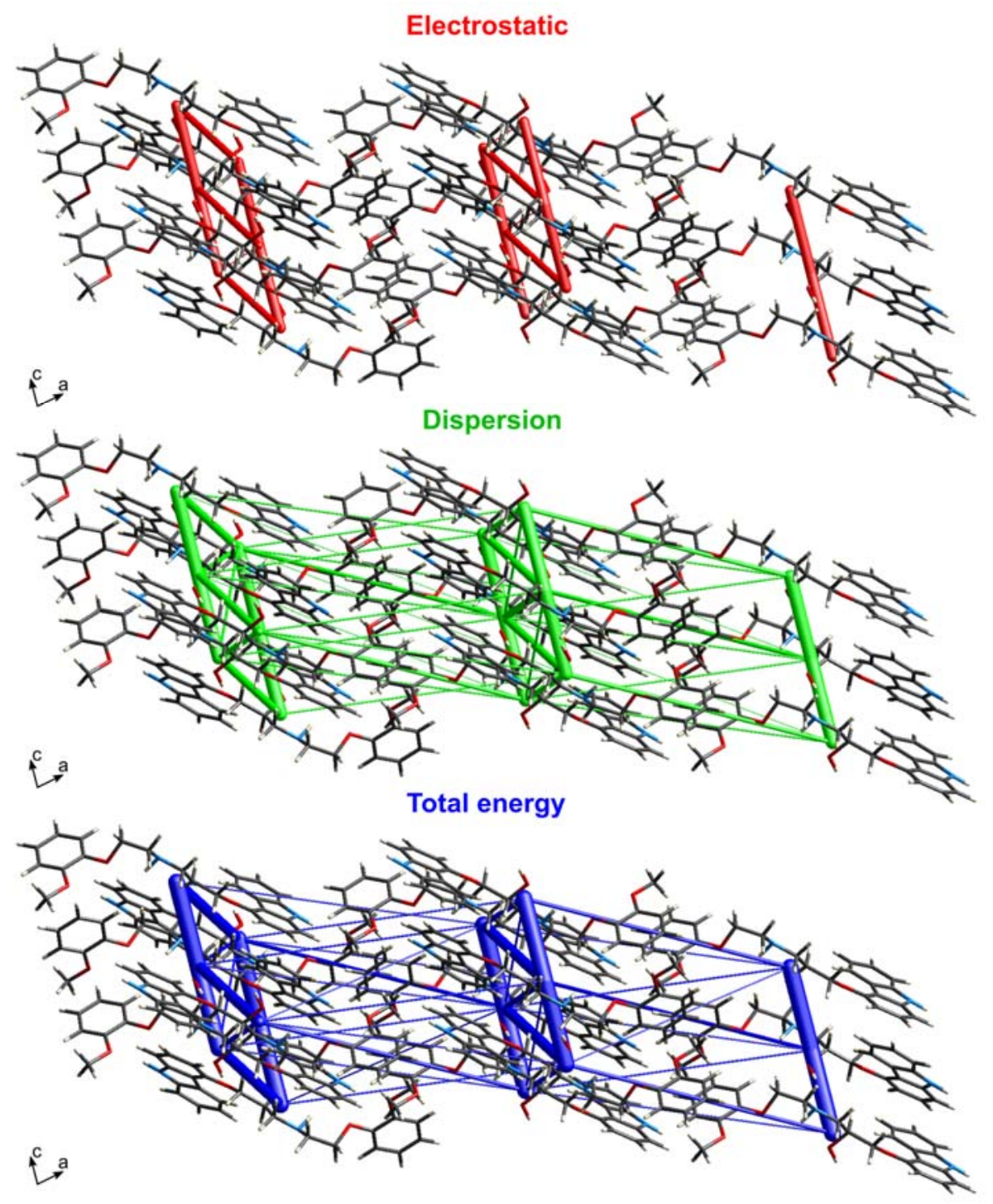

Figure S5. Energy framework diagrams for a cluster of nearest-neighbor molecules in carvedilol (form II). All diagrams use the same tube/cylinder scale of 50, and energies with a magnitude smaller than 5 $\mathrm{kJ} / \mathrm{mol}$ have been omitted for clarity. 
Table S1. Vibrational mode assignment for the calculated LFR spectrum of benzoic acid.

\begin{tabular}{|c|c|c|c|c|}
\hline Wavenumber, $\mathrm{cm}^{-1}$ & Symmetry & Relative activity & Mode type & Principal axis \\
\hline 31.06 & $\mathrm{~B}_{\mathrm{g}}$ & 745.04 & Translation & $\mathrm{ca}$ \\
\hline 48.93 & $\mathrm{~A}_{\mathrm{g}}$ & 311.88 & Translation & $\mathrm{b}$ \\
\hline 58.32 & $\mathrm{~A}_{\mathrm{g}}$ & 137.2 & Translation & $\mathrm{cb}$ \\
\hline 59.16 & $\mathrm{~B}_{\mathrm{g}}$ & 9.27 & Translation & $\mathrm{cb}$ \\
\hline 83.52 & $\mathrm{~A}_{\mathrm{g}}$ & 1000 & Torsion & $\mathrm{a}$ \\
\hline 84.61 & $\mathrm{~B}_{\mathrm{g}}$ & 365.15 & Torsion & $\mathrm{a}$ \\
\hline 97.71 & $\mathrm{~A}_{\mathrm{g}}$ & 685.82 & Ring torsion & $\mathrm{bc}$ \\
\hline 101.45 & $\mathrm{~B}_{\mathrm{g}}$ & 309.98 & Ring torsion & $\mathrm{bc}$ \\
\hline 121.48 & $\mathrm{~A}_{\mathrm{g}}$ & 273.37 & Ring torsion & $\mathrm{a}$ \\
\hline 124.81 & $\mathrm{~B}_{\mathrm{g}}$ & 56.4 & Ring torsion & $\mathrm{a}$ \\
\hline 126.16 & $\mathrm{~A}_{\mathrm{g}}$ & 841.83 & Ring torsion & $\mathrm{a}$ \\
\hline 127.76 & $\mathrm{~B}_{\mathrm{g}}$ & 3.72 & Carboxylic torsion & $\mathrm{c}$ \\
\hline 135.04 & $\mathrm{~A}_{\mathrm{g}}$ & 111.63 & Carboxylic torsion & $\mathrm{c}$ \\
\hline 135.54 & $\mathrm{~B}_{\mathrm{g}}$ & 55.62 & Carboxylic torsion & $\mathrm{c}$ \\
\hline 195.73 & $\mathrm{~A}_{\mathrm{g}}$ & 223.81 & Complex torsion & $\mathrm{ab}$ \\
\hline 196.44 & $\mathrm{~B}_{\mathrm{g}}$ & 2.62 & Complex torsion & $\mathrm{ab}$ \\
\hline 264.82 & $\mathrm{~B}_{\mathrm{g}}$ & 2.43 & Complex torsion & $\mathrm{c}$ \\
\hline 266.20 & $\mathrm{~A}_{\mathrm{g}}$ & 6.16 & Complex torsion & $\mathrm{c}$ \\
\hline
\end{tabular}
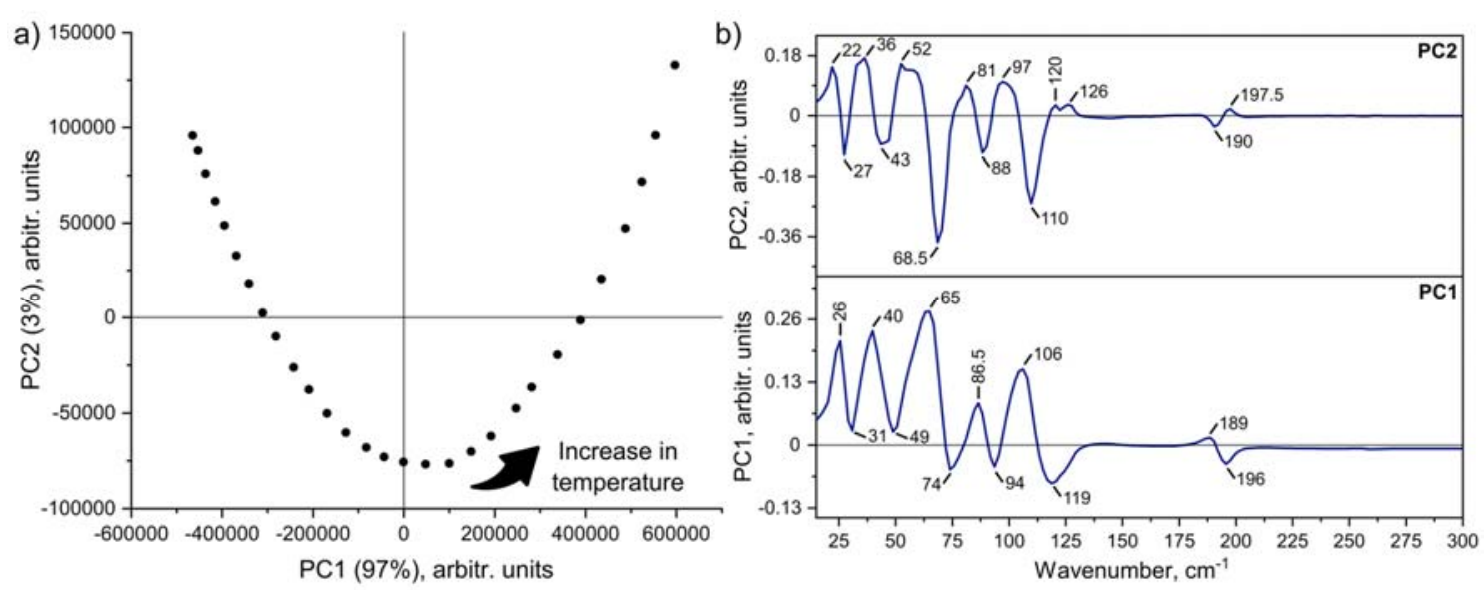

Figure S6. (a) Scores plot of PC1 versus PC2 and (b) the first two loadings plots from principal component analysis (PCA) of the variable temperature low-frequency Raman spectra of benzoic acid. 


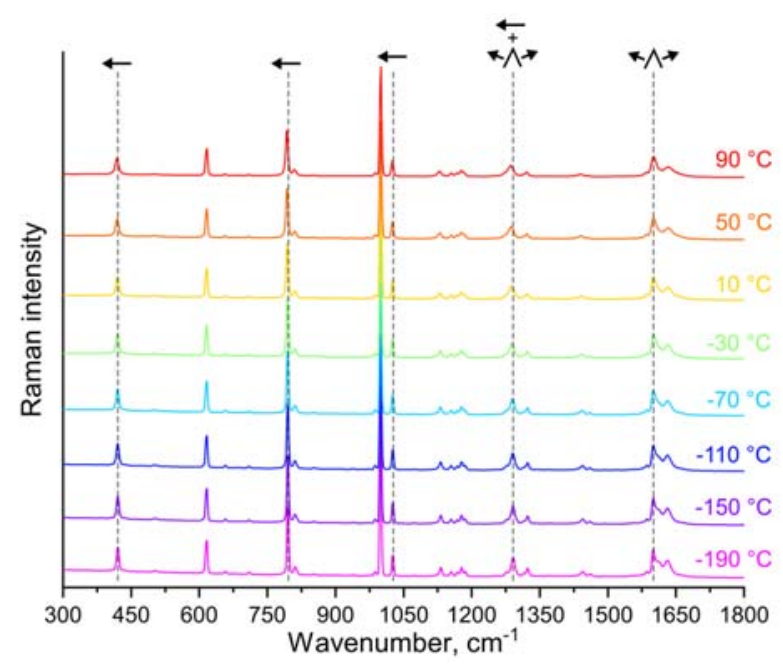

Figure S7. Mid-frequency Raman spectra of benzoic acid in a broad temperature range.
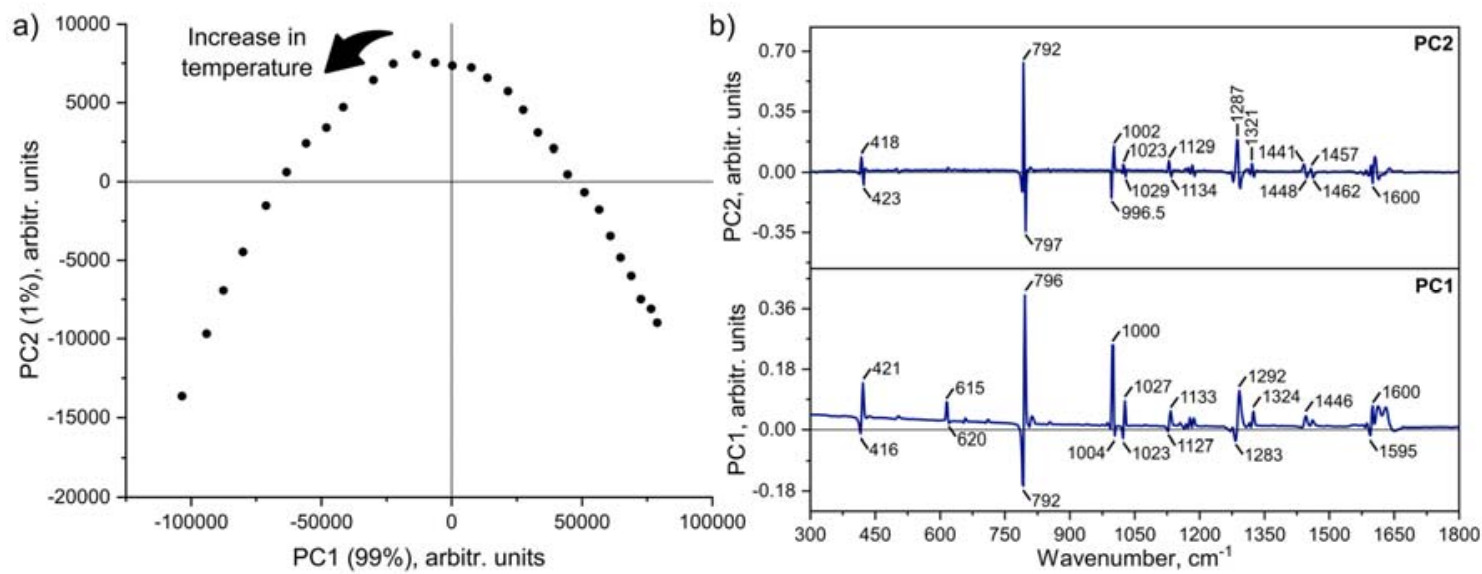

Figure S8. (a) Scores plot of PC1 versus PC2 and (b) the first two loadings plots from principal component analysis (PCA) of the variable temperature mid-frequency Raman spectra of benzoic acid. 

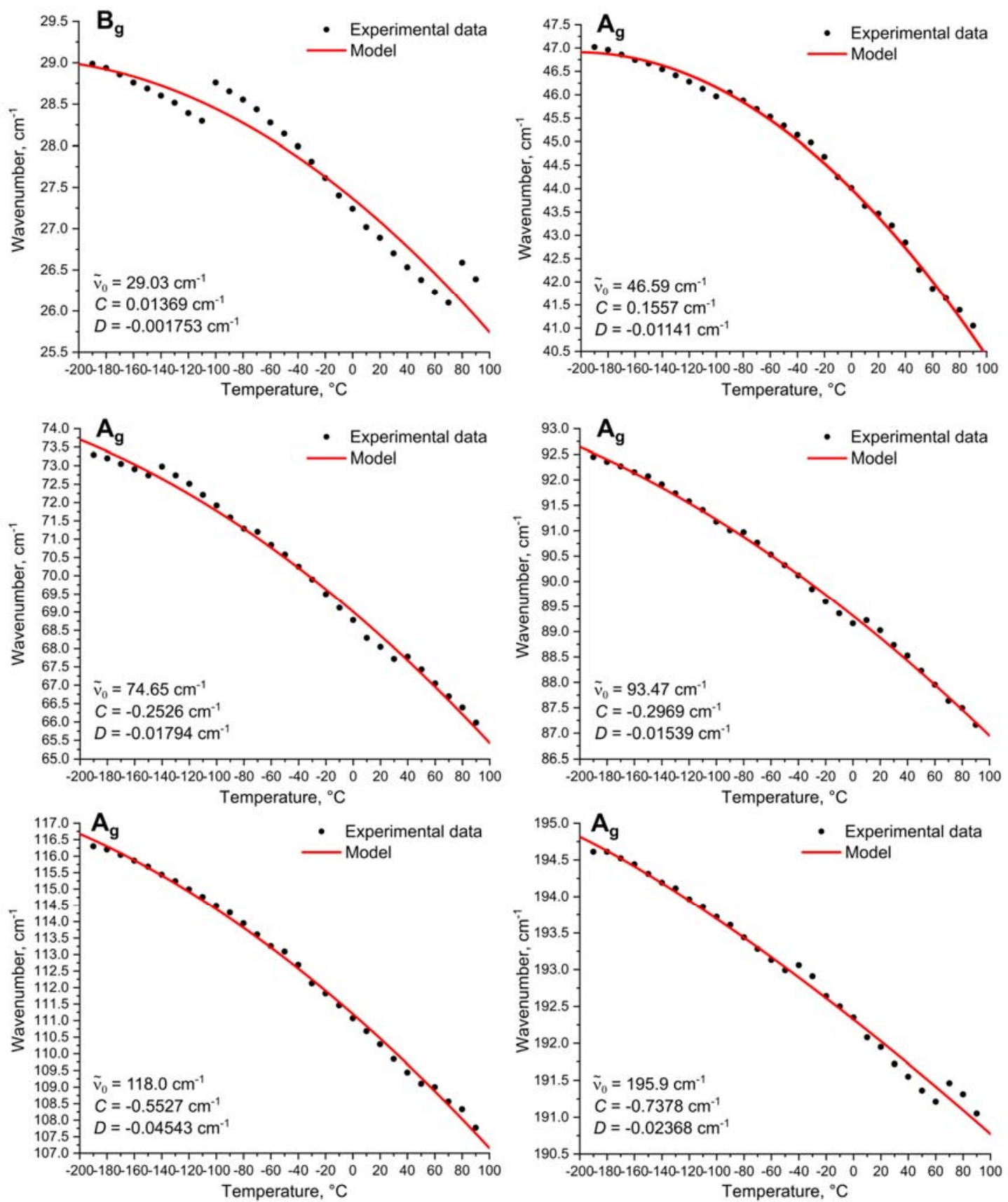

Figure S9. The modelling of temperature dependence of individual LFR peaks of benzoic acid as compared to the experimental results. 
Table S2. Vibrational mode assignment for the calculated LFR spectrum of diphenylacetic acid.

\begin{tabular}{|c|c|c|c|c|}
\hline Wavenumber, $\mathrm{cm}^{-1}$ & Symmetry & $\begin{array}{l}\text { Relative } \\
\text { activity }\end{array}$ & Mode type & Principal axis \\
\hline 33.81 & $\mathrm{~B}_{\mathrm{g}}$ & 158.1 & Translation & $\mathrm{b}$ \\
\hline 34.60 & $\mathrm{~A}_{\mathrm{g}}$ & 869.43 & Translation & $\mathrm{a}$ \\
\hline 40.49 & $\mathrm{~B}_{\mathrm{g}}$ & 582.96 & Translation & $\mathrm{a}$ \\
\hline 43.68 & $\overline{A_{g}}$ & 445.73 & Translation & $\mathrm{a}$ \\
\hline 55.78 & $\mathrm{~B}_{\mathrm{g}}$ & 13.15 & Translation & $\mathrm{b}$ \\
\hline 56.71 & $\overline{A_{g}}$ & 165.03 & Torsion & $\mathrm{c}$ \\
\hline 63.10 & $\mathrm{~B}_{\mathrm{g}}$ & 75.31 & Ring torsion & $\mathrm{ab}$ \\
\hline 68.68 & $\mathrm{~A}_{\mathrm{g}}$ & 126.05 & Ring torsion & $\mathrm{ab}$ \\
\hline 69.40 & $\mathrm{~B}_{\mathrm{g}}$ & 177.25 & Complex torsion & $\mathrm{ca}$ \\
\hline 75.57 & $\mathrm{~A}_{\mathrm{g}}$ & 746.82 & Complex torsion & $\mathrm{ab}$ \\
\hline 85.50 & $\mathrm{~A}_{\mathrm{g}}$ & 938.05 & Complex torsion & $\mathrm{bc}$ \\
\hline 86.44 & $\mathrm{~B}_{\mathrm{g}}$ & 239.49 & Complex torsion & $\mathrm{abc}$ \\
\hline 88.15 & $\mathrm{~A}_{\mathrm{g}}$ & 38.45 & Complex torsion & $\mathrm{bc}$ \\
\hline 92.30 & $\mathrm{~B}_{\mathrm{g}}$ & 847.13 & Complex torsion & $a b c$ \\
\hline 104.16 & $\mathrm{~A}_{\mathrm{g}}$ & 1000 & Complex torsion & acb \\
\hline 107.16 & $\overline{A_{g}}$ & 557.7 & Complex torsion & cba \\
\hline 107.67 & $\mathrm{~B}_{\mathrm{g}}$ & 336.22 & Complex torsion & cba \\
\hline 110.64 & $\mathrm{~B}_{\mathrm{g}}$ & 63.97 & Complex torsion & $\mathrm{cab}$ \\
\hline 140.85 & $\mathrm{~B}_{\mathrm{g}}$ & 37.09 & $\begin{array}{l}\text { Complex torsion (primarily } \\
\text { carboxylic) }\end{array}$ & $\mathrm{a}$ \\
\hline 142.73 & $\mathrm{~A}_{\mathrm{g}}$ & 338.55 & $\begin{array}{l}\text { Complex torsion (primarily } \\
\text { carboxylic) }\end{array}$ & $a b$ \\
\hline 164.43 & $\mathrm{~A}_{\mathrm{g}}$ & 122.11 & Complex torsion & $\mathrm{cab}$ \\
\hline 166.14 & $\overline{\mathrm{B}_{\mathrm{g}}}$ & 0.94 & Complex torsion & $\mathrm{cab}$ \\
\hline 179.07 & $\overline{\mathrm{B}_{\mathrm{g}}}$ & 3.65 & Complex torsion & $a b$ \\
\hline 183.63 & $\mathrm{~A}_{\mathrm{g}}$ & 48.71 & Complex torsion & $\mathrm{ab}$ \\
\hline 237.48 & $\mathrm{~B}_{\mathrm{g}}$ & 27.69 & Complex torsion (primarily ring) & acb \\
\hline 237.98 & $\mathrm{~A}_{\mathrm{g}}$ & 54.09 & Complex torsion (primarily ring) & acb \\
\hline 255.28 & $\mathrm{~A}_{\mathrm{g}}$ & 13.61 & Complex torsion & $\mathrm{ab}$ \\
\hline 257.16 & $\mathrm{~B}_{\mathrm{g}}$ & 6.71 & Complex torsion & $\mathrm{ab}$ \\
\hline 281.27 & $\mathrm{~B}_{\mathrm{g}}$ & 3.02 & Complex torsion (primarily ring) & acb \\
\hline 281.95 & $\overline{A_{g}}$ & 43.07 & Complex torsion (primarily ring) & $\mathrm{acb}$ \\
\hline
\end{tabular}



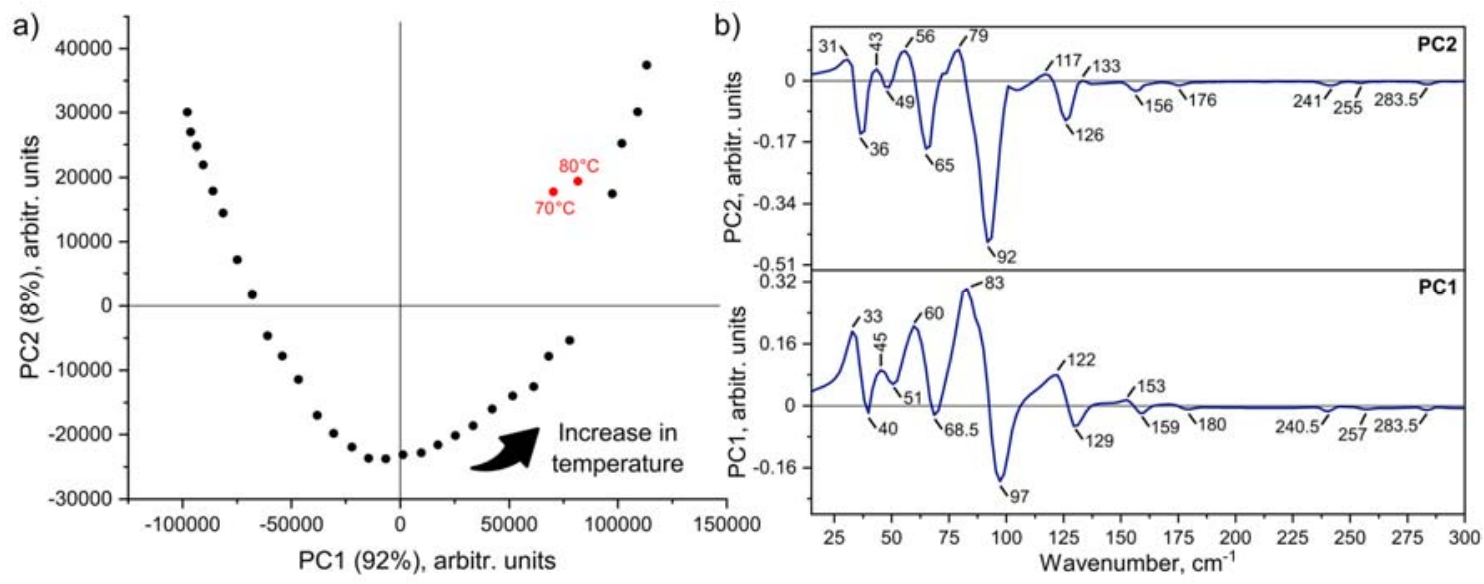

Figure S10. (a) Scores plot of PC1 versus PC2 and (b) the first two loadings plots from principal component analysis (PCA) of the variable temperature low-frequency Raman spectra of diphenylacetic acid.

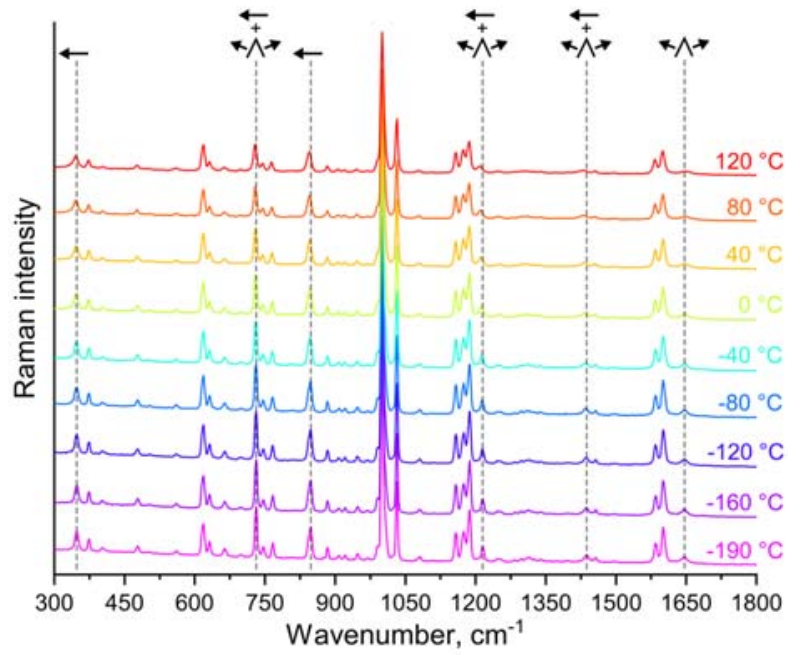

Figure S11. Mid-frequency Raman spectra of diphenylacetic acid in a broad temperature range.
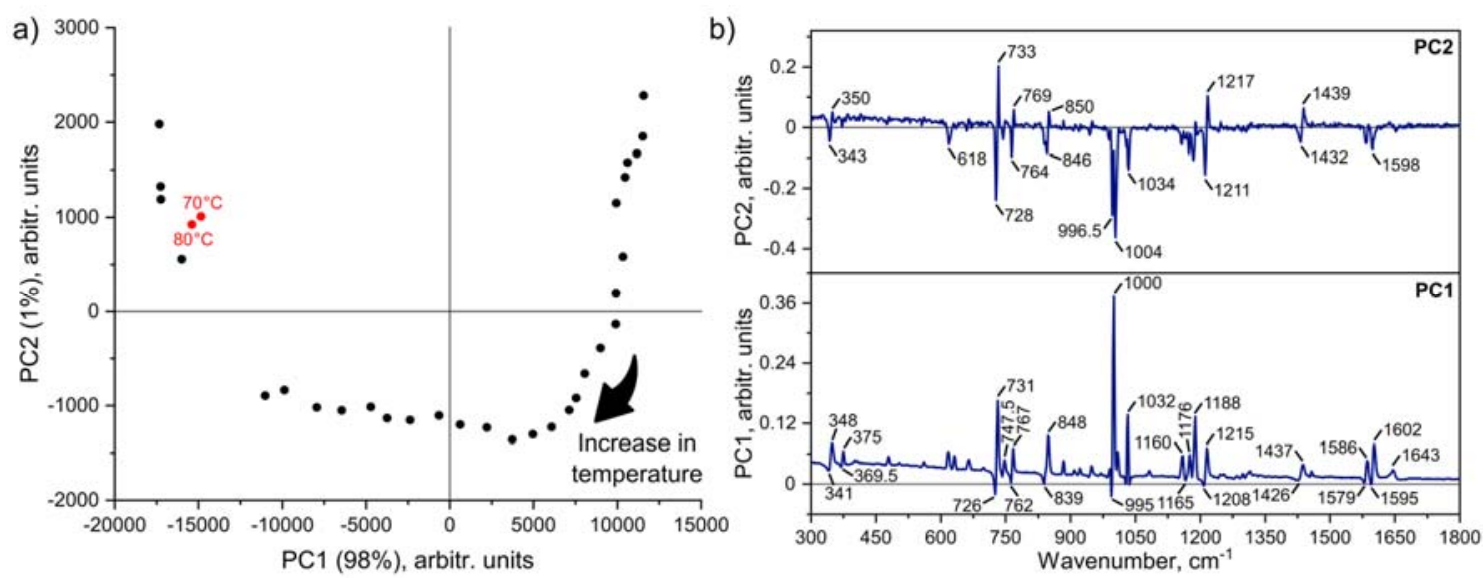

Figure S12. (a) Scores plot of PC1 versus PC2 and (b) the first two loadings plots from principal component analysis (PCA) of the variable temperature mid-frequency Raman spectra of diphenylacetic acid. 

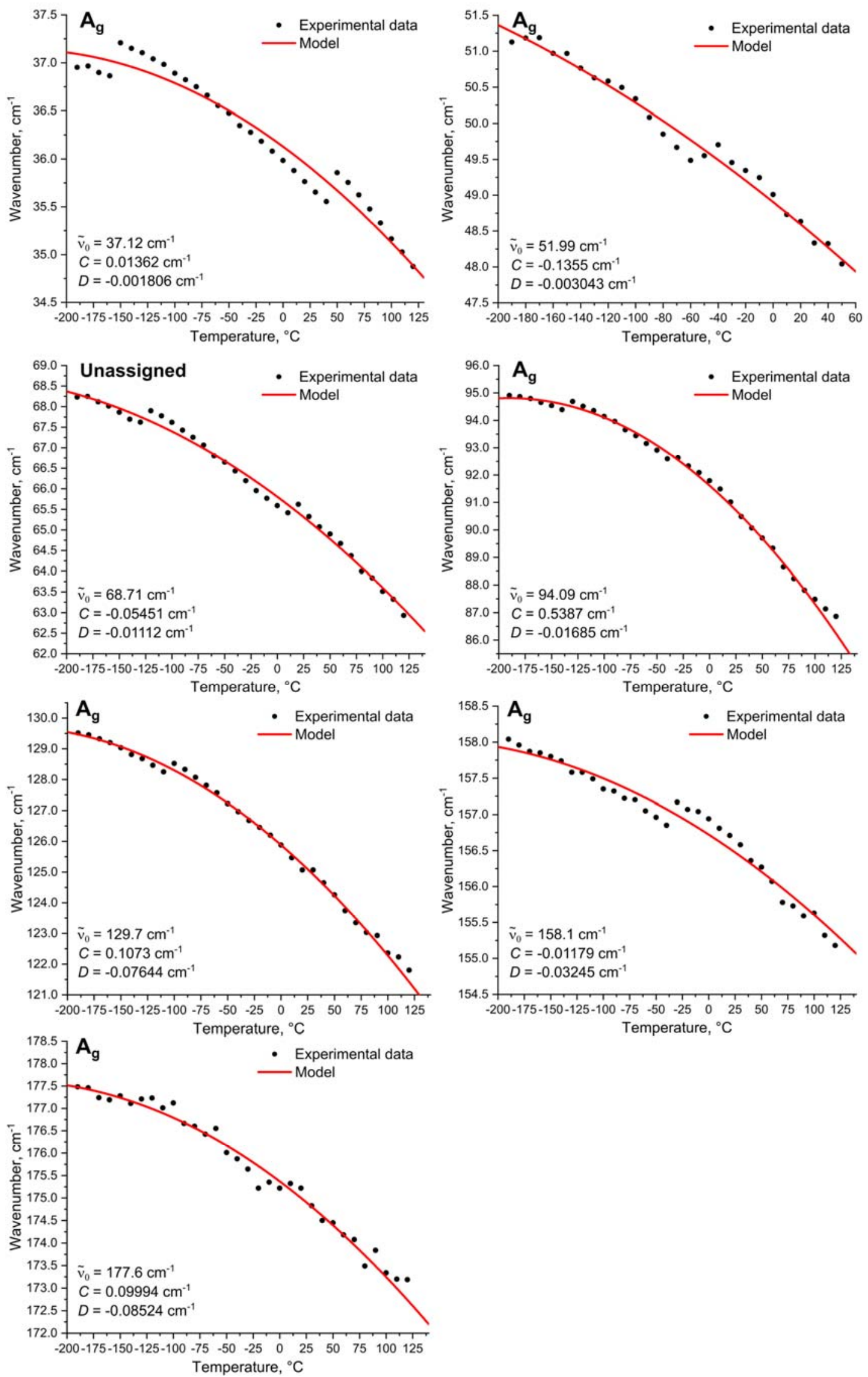
Figure S13. The modelling of temperature dependence of individual LFR peaks of diphenylacetic acid as compared to the experimental results. A limited range of fitted data is shown for the band at $\sim 51 \mathrm{~cm}^{-}$ ${ }^{1}\left(-190^{\circ} \mathrm{C}\right)$ due to its severe broadening at higher temperatures.

Table S3. Vibrational mode assignment for the calculated LFR spectrum of L-tyrosine.

\begin{tabular}{|c|c|c|c|c|}
\hline Wavenumber, $\mathrm{cm}^{-1}$ & Symmetry & $\begin{array}{l}\text { Relative } \\
\text { activity }\end{array}$ & Mode type & Principal axis \\
\hline 27.97 & A & 1000 & Translation & $\mathrm{a}$ \\
\hline 31.43 & $\mathrm{~B}_{3}$ & 4.87 & Translation & $\mathrm{c}$ \\
\hline 36.31 & A & 39.51 & Translation & $\mathrm{c}$ \\
\hline 54.90 & $\mathrm{~B}_{3}$ & 2.03 & Translation & $\mathrm{a}$ \\
\hline 56.65 & $\mathrm{~B}_{2}$ & 49.93 & Translation & $\mathrm{c}$ \\
\hline 66.01 & $\mathrm{~B}_{2}$ & 1.34 & Translation & $\mathrm{b}$ \\
\hline 74.75 & A & 164.6 & Torsion & ba \\
\hline 75.63 & $\mathrm{~B}_{1}$ & 63.16 & Torsion & ba \\
\hline 86.26 & $\mathrm{~B}_{2}$ & 78.89 & Torsion & $\mathrm{a}$ \\
\hline 87.39 & $\mathrm{~B}_{3}$ & 42.7 & Torsion & ac \\
\hline 91.23 & $\mathrm{~B}_{2}$ & 0.06 & Torsion & $\mathrm{ac}$ \\
\hline 95.34 & $\mathrm{~B}_{1}$ & 269.87 & Torsion & ba \\
\hline 102.14 & $\mathrm{~B}_{1}$ & 372.49 & Torsion & $\mathrm{ab}$ \\
\hline 115.55 & $\mathrm{~B}_{3}$ & 4.27 & Torsion & ba \\
\hline 116.62 & A & 679.45 & Complex torsion & $a b c$ \\
\hline 119.16 & $\bar{A}$ & 793.44 & Torsion & $\mathrm{ab}$ \\
\hline 122.33 & $\mathrm{~B}_{2}$ & 112.57 & $\begin{array}{l}\text { Complex torsion (primarily } \\
\text { carboxylic) }\end{array}$ & ba \\
\hline 122.96 & $\mathrm{~B}_{1}$ & 25.31 & Complex torsion (primarily ring) & $\mathrm{ac}$ \\
\hline 124.04 & A & 44.36 & Complex torsion & $\mathrm{ab}$ \\
\hline 125.68 & $\mathrm{~B}_{1}$ & 214.61 & Complex torsion & abc \\
\hline 131.09 & $\mathrm{~B}_{1}$ & 116.24 & Complex torsion & $\mathrm{ab}$ \\
\hline 132.44 & A & 216.71 & $\begin{array}{l}\text { Complex torsion (primarily } \\
\text { carboxylic) }\end{array}$ & bc \\
\hline 135.49 & $\mathrm{~B}_{3}$ & 3.65 & $\begin{array}{l}\text { Complex torsion (primarily } \\
\text { carboxylic) }\end{array}$ & $a b$ \\
\hline 141.95 & $\mathrm{~B}_{2}$ & 22.31 & Complex torsion & $\mathrm{ab}$ \\
\hline 143.07 & A & 17.23 & Complex torsion & bca \\
\hline 147.06 & $\mathrm{~B}_{1}$ & 62.21 & Complex torsion & $\mathrm{ab}$ \\
\hline 152.10 & $\mathrm{~B}_{3}$ & 30.2 & $\begin{array}{c}\text { Complex torsion (primarily } \\
\text { carboxylic) }\end{array}$ & ba \\
\hline
\end{tabular}




\begin{tabular}{|c|c|c|c|c|}
\hline 153.08 & $\mathrm{~B}_{2}$ & 0.07 & $\begin{array}{l}\text { Complex torsion (primarily } \\
\text { carboxylic) }\end{array}$ & ba \\
\hline 157.75 & $\mathrm{~B}_{1}$ & 38.83 & Complex torsion & $\mathrm{ca}$ \\
\hline 161.19 & $\mathrm{~B}_{3}$ & 676.96 & Complex torsion & $\mathrm{ab}$ \\
\hline 163.18 & $\mathrm{~B}_{2}$ & 38.48 & Complex torsion (primarily ring) & $\mathrm{ab}$ \\
\hline 164.22 & $\mathrm{~B}_{3}$ & 447.87 & Complex torsion & $\mathrm{ab}$ \\
\hline 165.47 & $\mathrm{~A}$ & 52.51 & Complex torsion & $\mathrm{cb}$ \\
\hline 173.34 & A & 156.24 & Complex torsion & $\mathrm{ab}$ \\
\hline 186.46 & $\mathrm{~B}_{3}$ & 105.17 & Complex torsion & $\mathrm{ca}$ \\
\hline 189.32 & $\mathrm{~B}_{1}$ & 5.4 & Complex torsion & $\mathrm{ab}$ \\
\hline 195.74 & $\mathrm{~B}_{2}$ & 0.62 & Complex torsion & $\mathrm{bc}$ \\
\hline 210.52 & A & 19.29 & $\begin{array}{l}\text { Complex torsion (primarily } \\
\text { amino groups) }\end{array}$ & ba \\
\hline 218.68 & $\mathrm{~B}_{2}$ & 0.71 & $\begin{array}{l}\text { Complex torsion (primarily } \\
\text { amino groups) }\end{array}$ & $\mathrm{bc}$ \\
\hline 224.63 & $\mathrm{~B}_{3}$ & 7.37 & $\begin{array}{c}\text { Complex torsion (primarily } \\
\text { amino groups) }\end{array}$ & bc \\
\hline 238.11 & $\mathrm{~B}_{1}$ & 0.31 & $\begin{array}{l}\text { Complex torsion (primarily } \\
\text { amino groups) }\end{array}$ & ba \\
\hline 248.97 & $\mathrm{~B}_{3}$ & 0.05 & $\begin{array}{l}\text { Complex torsion (primarily } \\
\text { amino groups) }\end{array}$ & ba \\
\hline 259.56 & $\mathrm{~A}$ & 53.04 & Complex torsion & $\mathrm{ab}$ \\
\hline 260.33 & $\mathrm{~B}_{1}$ & 30.97 & Complex torsion & ba \\
\hline
\end{tabular}



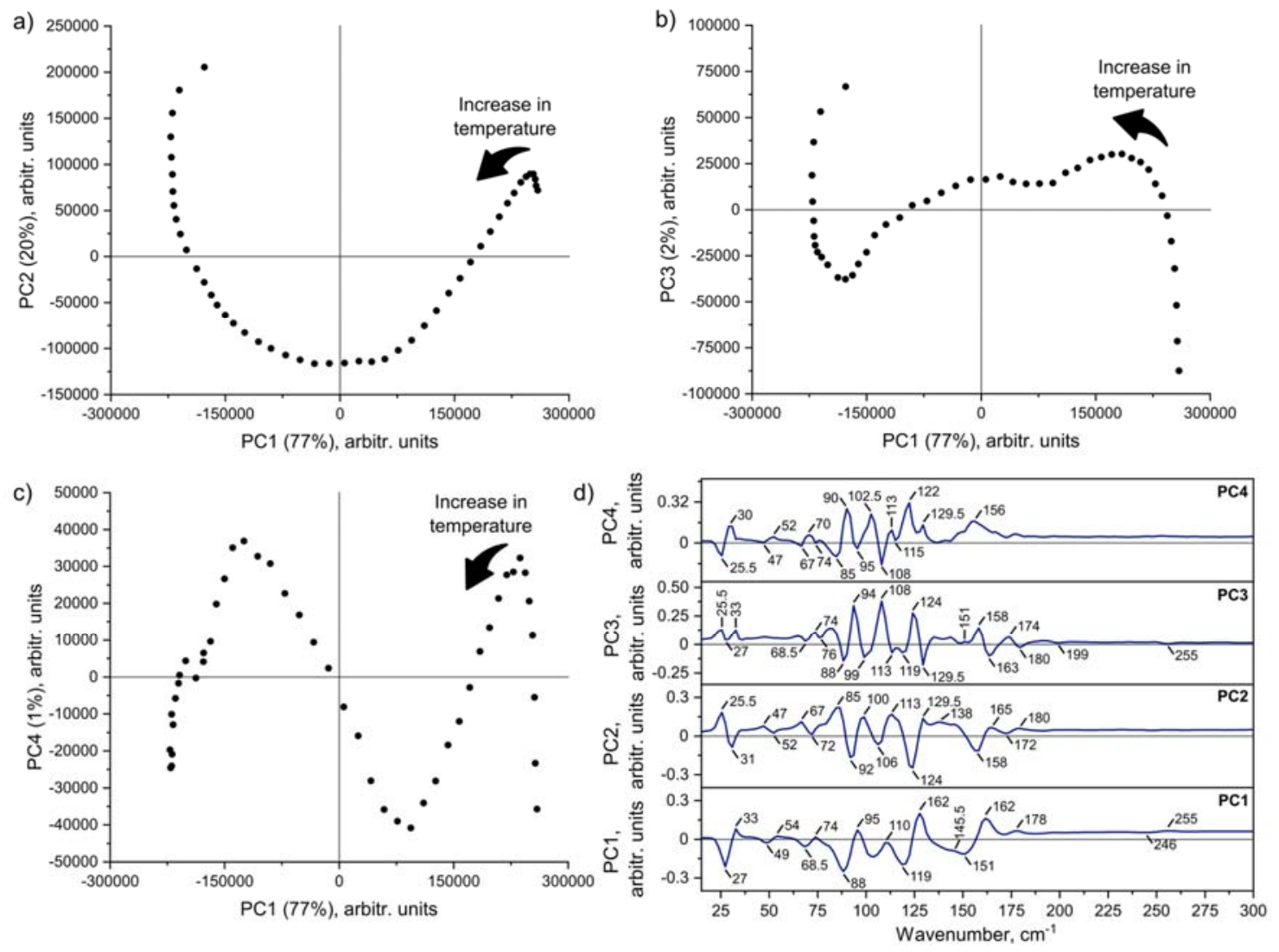

Figure S14. Scores plot of (a) PC1 versus PC2, (b) PC1 versus PC3, (c) PC1 versus PC4 and (d) the first four loadings plots from principal component analysis (PCA) of the variable temperature lowfrequency Raman spectra of L-tyrosine.

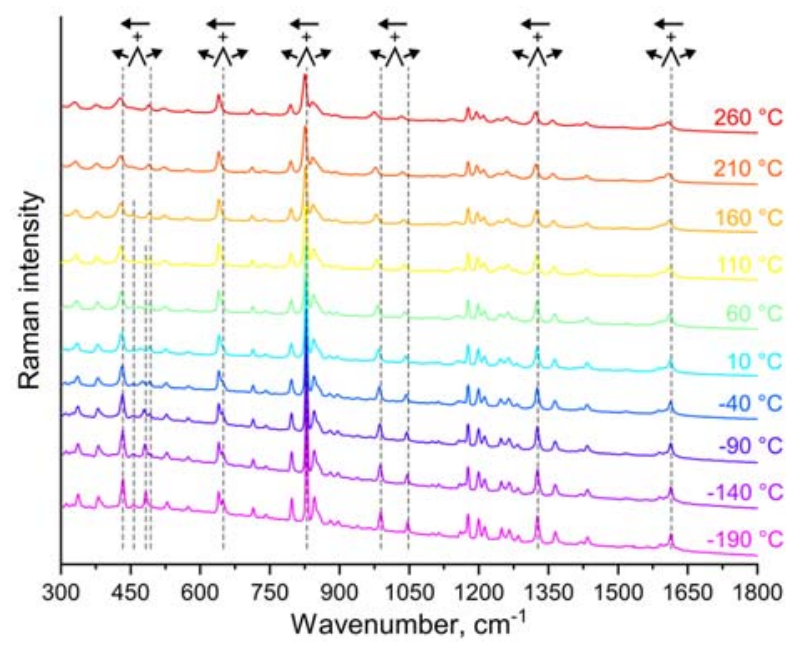

Figure S15. Mid-frequency Raman spectra of L-tyrosine in a broad temperature range. 

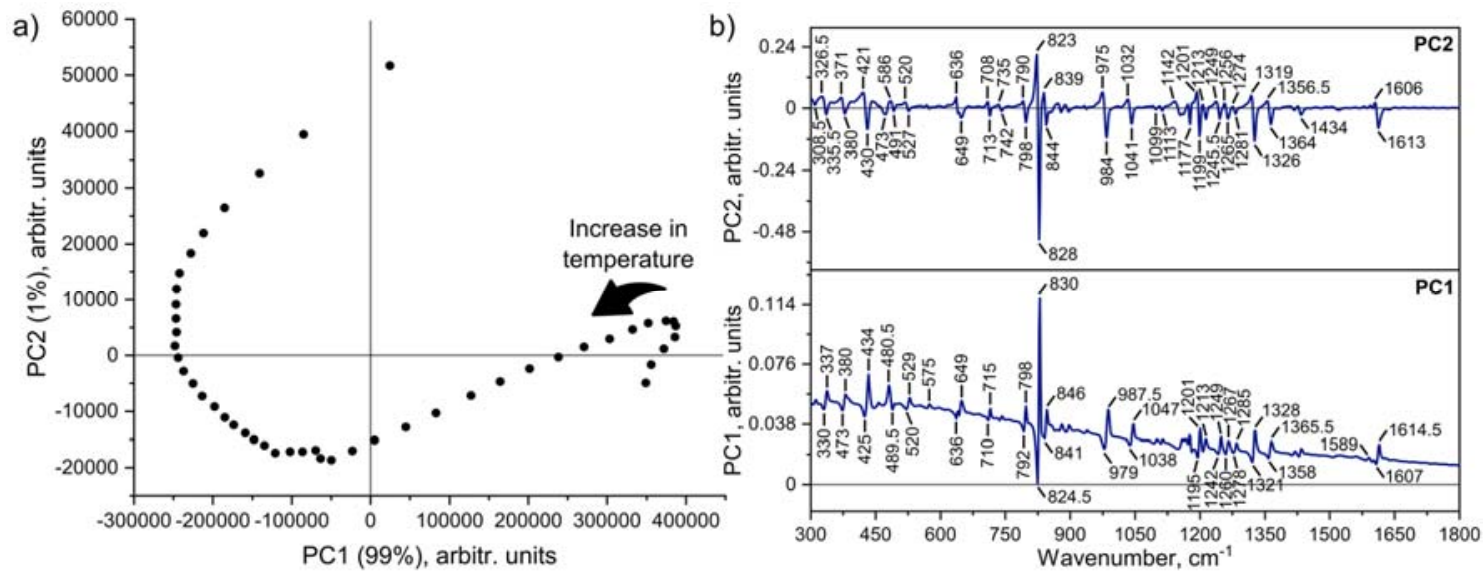

Figure S16. (a) Scores plot of PC1 versus PC2 and (b) the first two loadings plots from principal component analysis (PCA) of the variable temperature mid-frequency Raman spectra of L-tyrosine. 

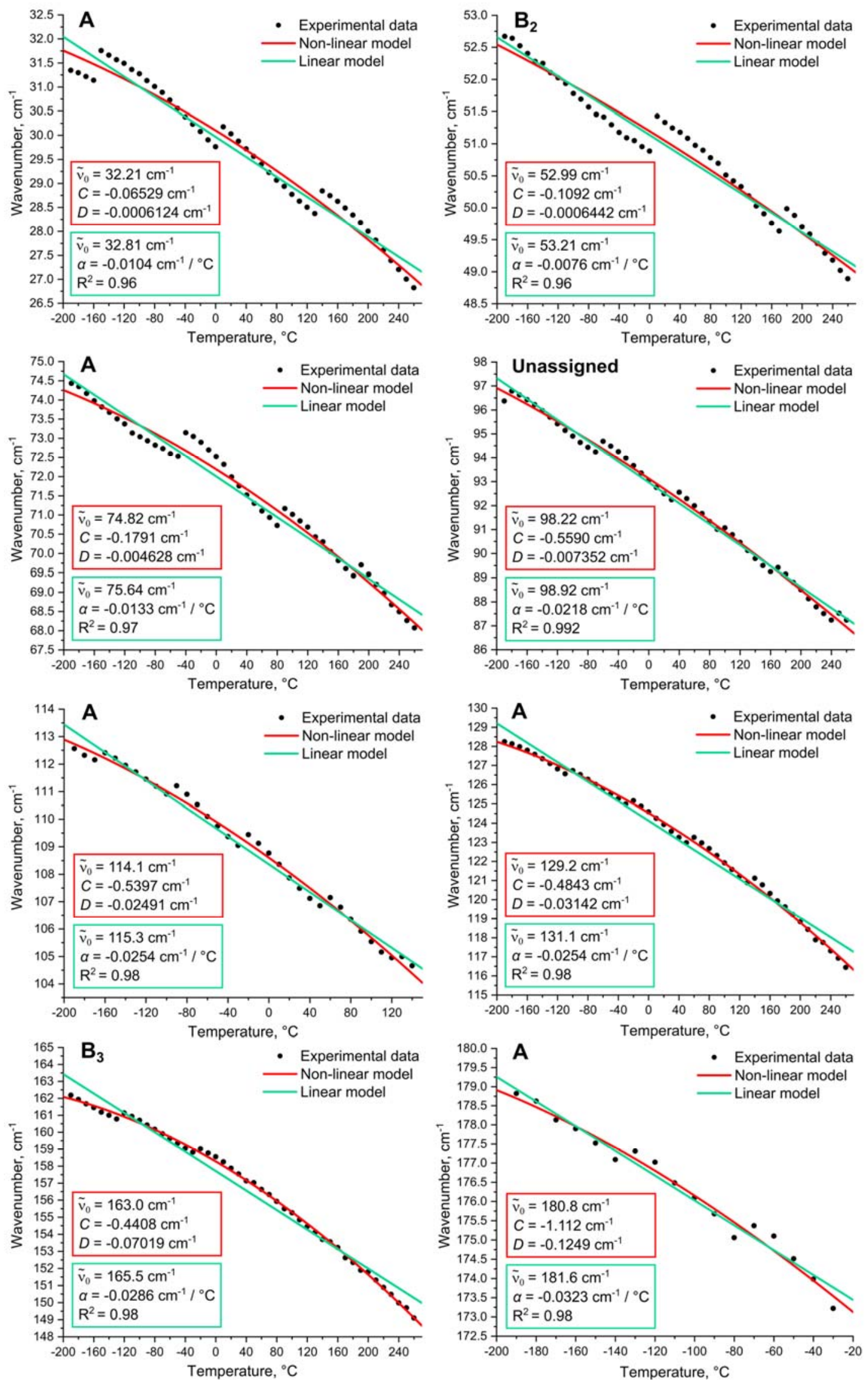
Figure S17. The modelling of temperature dependence of individual LFR peaks of L-tyrosine as compared to the experimental results. A very limited range of fitted data is shown for the band at $\sim 179$ $\mathrm{cm}^{-1}\left(-190{ }^{\circ} \mathrm{C}\right)$ due to its severe broadening at higher temperatures.

Table S4. Vibrational mode assignment for the calculated LFR spectrum of celecoxib (form III).

\begin{tabular}{|c|c|c|c|c|}
\hline Wavenumber, $\mathrm{cm}^{-1}$ & Symmetry & $\begin{array}{l}\text { Relative } \\
\text { activity }\end{array}$ & Mode type & Principal axis \\
\hline 27.96 & \multirow{23}{*}{$\mathrm{A}_{\mathrm{g}}$} & 202.71 & $\begin{array}{c}\text { Translation + Methyl group } \\
\text { rotation }\end{array}$ & $\mathrm{b}$ \\
\hline 29.59 & & 373.92 & Translation & $\mathrm{b}$ \\
\hline 46.12 & & 127.5 & $\begin{array}{c}\text { Complex torsion + Methyl group } \\
\text { rotation }\end{array}$ & $\mathrm{cb}$ \\
\hline 51.15 & & 401.31 & $\begin{array}{l}\text { Complex torsion (primarily } \\
\text { trifluoromethyl group) }\end{array}$ & $a b$ \\
\hline 51.82 & & 225.92 & Complex torsion & ac \\
\hline 59.83 & & 215.84 & Complex torsion & ac \\
\hline 65.51 & & 103.1 & Complex torsion & bc \\
\hline 74.53 & & 282.49 & Complex torsion & ba \\
\hline 81.35 & & 266.42 & $\begin{array}{c}\text { Complex torsion + Methyl group } \\
\text { rotation }\end{array}$ & bc \\
\hline 85.66 & & 221.45 & $\begin{array}{l}\text { Complex torsion (primarily p-tolyl } \\
\text { group) }\end{array}$ & bc \\
\hline 92.82 & & 183.49 & $\begin{array}{l}\text { Complex torsion (primarily p-tolyl } \\
\text { group) }\end{array}$ & ac \\
\hline 99.18 & & 388.18 & Complex torsion & $a b$ \\
\hline 113.56 & & 232.65 & Complex torsion (primarily ring) & $\mathrm{b}$ \\
\hline 126.14 & & 1000 & Complex torsion (primarily ring) & $\mathrm{bc}$ \\
\hline 132.10 & & 89.89 & Complex torsion (primarily ring) & $\mathrm{b}$ \\
\hline 141.17 & & 431.25 & $\begin{array}{l}\text { Complex torsion (primarily p-tolyl } \\
\text { group) }\end{array}$ & bc \\
\hline 159.76 & & 23.93 & $\begin{array}{l}\text { Complex torsion (primarily p-tolyl } \\
\text { group) }\end{array}$ & $\mathrm{bc}$ \\
\hline 177.51 & & 73.57 & Complex torsion & $a b$ \\
\hline 191.84 & & 23.6 & Complex torsion & $a b$ \\
\hline 201.04 & & 81.67 & Complex torsion & $\mathrm{a}$ \\
\hline 219.16 & & 21.72 & $\begin{array}{l}\text { Complex torsion + Methyl group } \\
\text { stretching }\end{array}$ & $a b c$ \\
\hline 245.01 & & 29.84 & Complex torsion & $\mathrm{ab}$ \\
\hline 252.93 & & 47.72 & Complex torsion & $\mathrm{b}$ \\
\hline
\end{tabular}



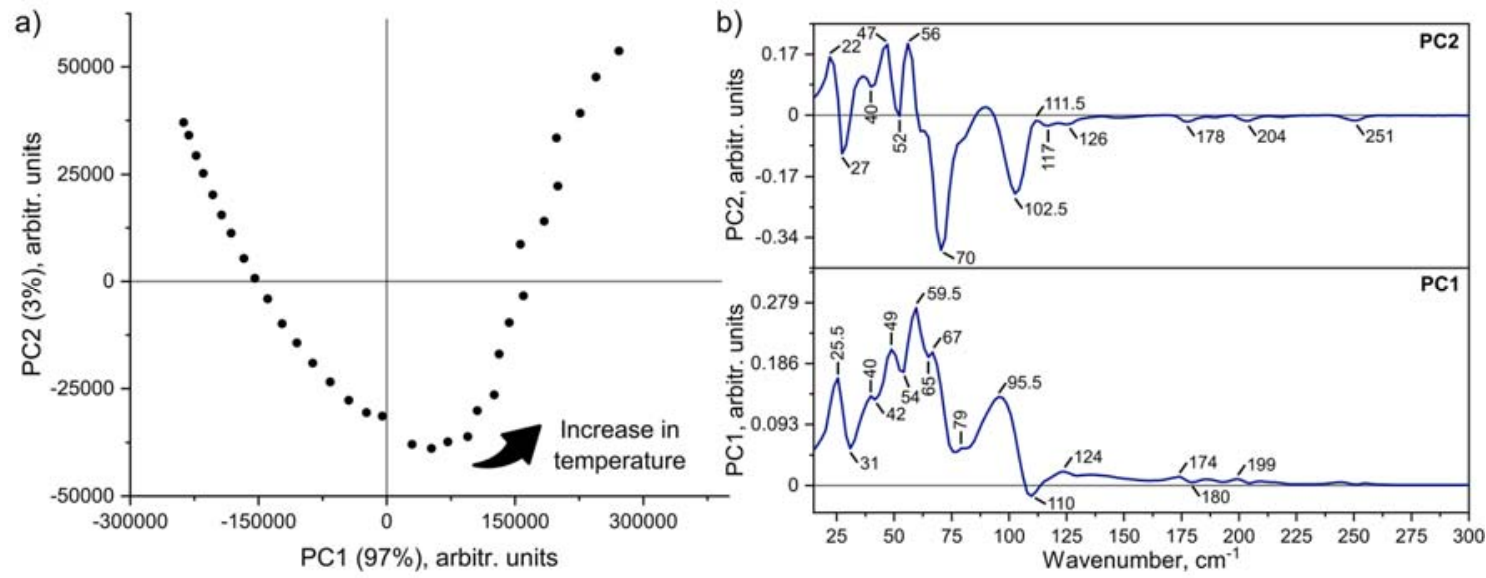

Figure S18. (a) Scores plot of PC1 versus PC2 and (b) the first two loadings plots from principal component analysis (PCA) of the variable temperature low-frequency Raman spectra of celecoxib (form III).

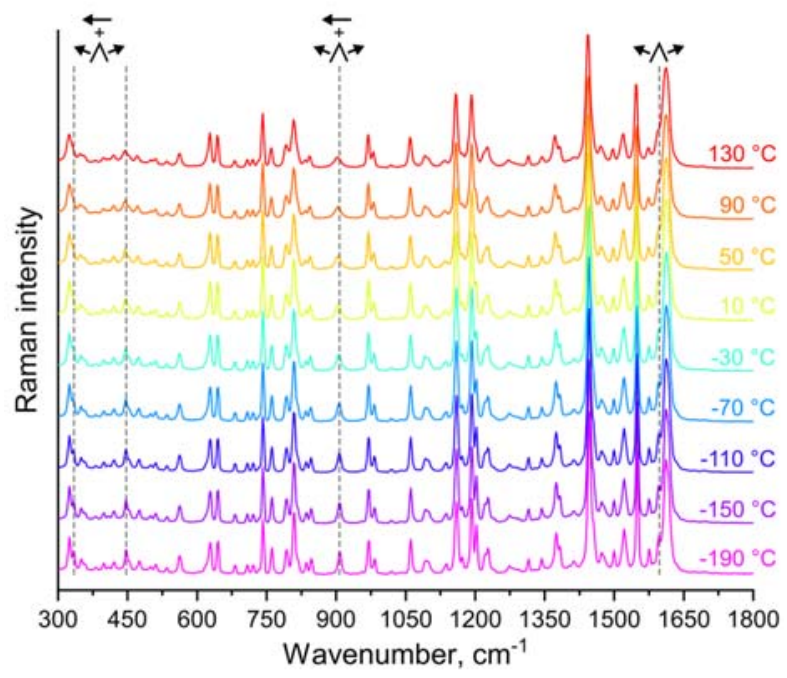

Figure S19. Mid-frequency Raman spectra of celecoxib (form III) in a broad temperature range.
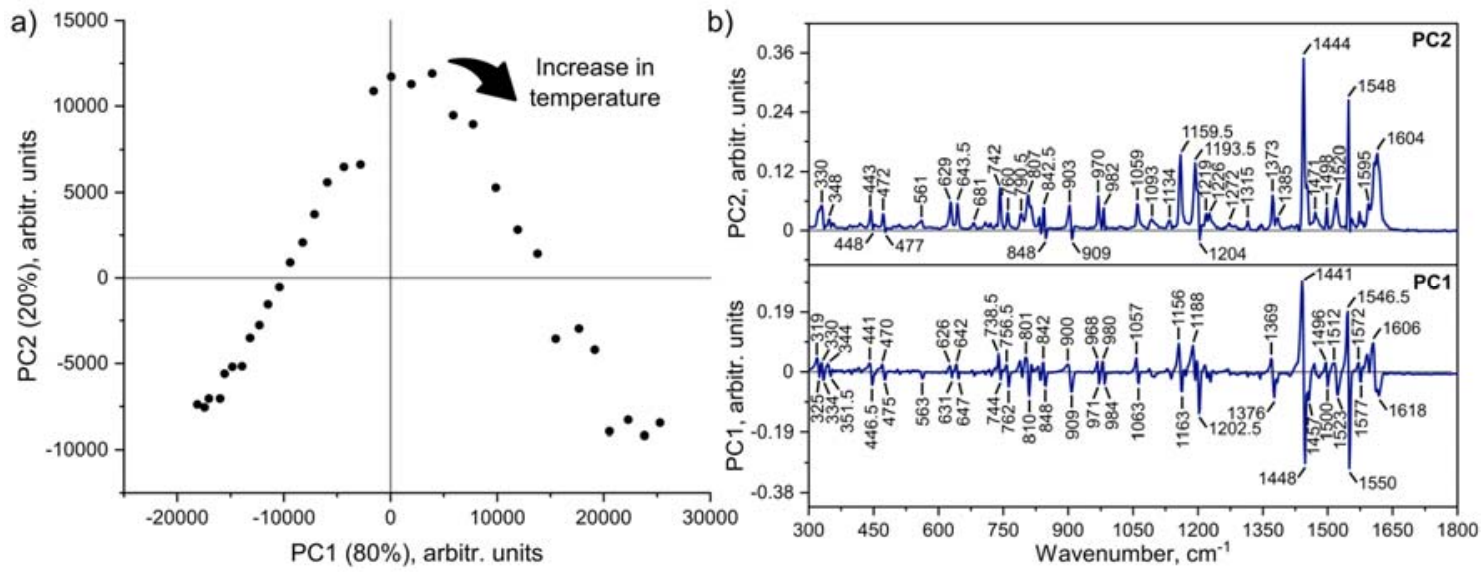

Figure S20. (a) Scores plot of PC1 versus PC2 and (b) the first two loadings plots from principal component analysis (PCA) of the variable temperature mid-frequency Raman spectra of celecoxib (form III). 

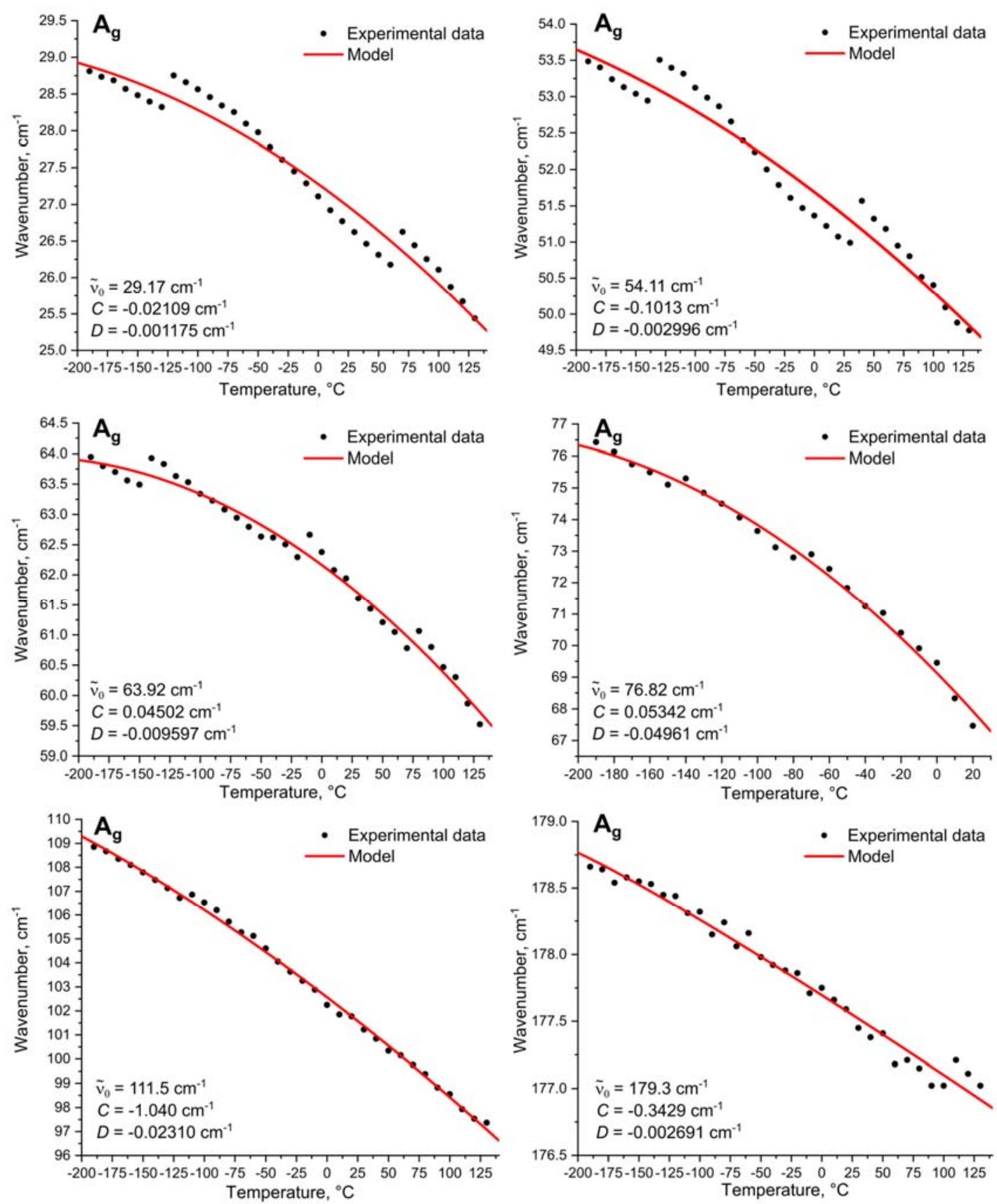

Figure S21. The modelling of temperature dependence of individual LFR peaks of celecoxib (form III) as compared to the experimental results. A limited range of fitted data is shown for the band at $\sim 77 \mathrm{~cm}^{-}$ ${ }^{1}\left(-190^{\circ} \mathrm{C}\right)$ due to its severe broadening at higher temperatures.

Table S5. Vibrational mode assignment for the calculated LFR spectrum of carvedilol (form II).

\begin{tabular}{|c|c|c|c|c|}
\hline Wavenumber, $\mathrm{cm}^{-1}$ & Symmetry & $\begin{array}{c}\text { Relative } \\
\text { activity }\end{array}$ & Mode type & Principal axis \\
\hline 20.78 & $\mathrm{~A}_{\mathrm{g}}$ & 842.37 & Translation & $\mathrm{b}$ \\
\hline 22.34 & $\mathrm{~B}_{\mathrm{g}}$ & 974.2 & Translation & $\mathrm{a}$ \\
\hline
\end{tabular}




\begin{tabular}{|c|c|c|c|c|}
\hline 27.94 & $\mathrm{~B}_{\mathrm{g}}$ & 20.75 & Translation & $\mathrm{b}$ \\
\hline 31.83 & $\mathrm{~B}_{\mathrm{g}}$ & 74.89 & Translation & $\mathrm{a}$ \\
\hline 35.95 & $\mathrm{~B}_{\mathrm{g}}$ & 461.53 & Complex torsion & bca \\
\hline 35.95 & $\mathrm{~A}_{\mathrm{g}}$ & 679.34 & Complex torsion & $\mathrm{bc}$ \\
\hline 43.28 & $\mathrm{~A}_{\mathrm{g}}$ & 1000 & Complex torsion & $\mathrm{bc}$ \\
\hline 44.28 & $\mathrm{~A}_{\mathrm{g}}$ & 408.03 & Complex torsion & $\mathrm{bc}$ \\
\hline 49.86 & $\mathrm{~A}_{\mathrm{g}}$ & 151.22 & Complex torsion & $\mathrm{a}$ \\
\hline 52.16 & $\mathrm{~B}_{\mathrm{g}}$ & 232.44 & Complex torsion & $\mathrm{bc}$ \\
\hline 55.30 & $\mathrm{~B}_{\mathrm{g}}$ & 12.84 & Complex torsion & ba \\
\hline 55.63 & $\mathrm{~A}_{\mathrm{g}}$ & 211.56 & Complex torsion & $\mathrm{bc}$ \\
\hline 57.20 & $\mathrm{~B}_{\mathrm{g}}$ & 40.91 & Complex torsion & $\mathrm{bc}$ \\
\hline 63.17 & $\mathrm{~A}_{\mathrm{g}}$ & 211.8 & Complex torsion & $\mathrm{bc}$ \\
\hline 69.08 & $\mathrm{~A}_{\mathrm{g}}$ & 215.91 & Complex torsion & $\mathrm{bc}$ \\
\hline 71.16 & $\mathrm{~B}_{\mathrm{g}}$ & 199.9 & Complex torsion & $\mathrm{bc}$ \\
\hline 79.98 & $\mathrm{~B}_{\mathrm{g}}$ & 556.1 & Complex torsion & $\mathrm{acb}$ \\
\hline 84.92 & $\mathrm{~A}_{\mathrm{g}}$ & 155.76 & Complex torsion (primarily ring) & acb \\
\hline 87.28 & $\mathrm{~A}_{\mathrm{g}}$ & 135.42 & Complex torsion & $\mathrm{a}$ \\
\hline 87.87 & $\mathrm{~B}_{\mathrm{g}}$ & 75.46 & Complex torsion & $\mathrm{b}$ \\
\hline 90.80 & $\mathrm{~B}_{\mathrm{g}}$ & 22.69 & Complex torsion & $\mathrm{ab}$ \\
\hline 94.80 & $\mathrm{~A}_{\mathrm{g}}$ & 466.13 & Complex torsion & $\mathrm{ab}$ \\
\hline 97.37 & $\mathrm{~B}_{\mathrm{g}}$ & 283.61 & Carbazole torsion & $\mathrm{a}$ \\
\hline 110.64 & $\mathrm{~B}_{\mathrm{g}}$ & 82.63 & Complex torsion & ba \\
\hline 111.97 & $\mathrm{~A}_{\mathrm{g}}$ & 573.83 & Complex torsion & $\mathrm{ac}$ \\
\hline 115.30 & $\mathrm{~A}_{\mathrm{g}}$ & 304.25 & Carbazole torsion & $\mathrm{ac}$ \\
\hline 118.88 & $\mathrm{~A}_{\mathrm{g}}$ & 29.99 & $\begin{array}{l}\text { Complex torsion (primarily } \\
\text { ether/alkyl chain) }\end{array}$ & $\mathrm{bc}$ \\
\hline 120.08 & $\mathrm{~B}_{\mathrm{g}}$ & 185.43 & $\begin{array}{l}\text { Complex torsion (primarily } \\
\text { ether/alkyl chain) }\end{array}$ & $\mathrm{bc}$ \\
\hline 124.07 & $\mathrm{~B}_{\mathrm{g}}$ & 115.47 & $\begin{array}{l}\text { Complex torsion (primarily } \\
\text { ether/alkyl chain) }\end{array}$ & $\mathrm{bc}$ \\
\hline 128.00 & $\mathrm{~A}_{\mathrm{g}}$ & 62.02 & $\begin{array}{l}\text { Complex torsion (primarily } \\
\text { ether/alkyl chain) }\end{array}$ & $\mathrm{bc}$ \\
\hline 128.51 & $\mathrm{~B}_{\mathrm{g}}$ & 209.71 & $\begin{array}{l}\text { Complex torsion (primarily } \\
\text { ether/alkyl chain) }\end{array}$ & $a b c$ \\
\hline 143.28 & $\mathrm{~B}_{\mathrm{g}}$ & 51.3 & Carbazole bending/torsion & $\mathrm{ca}$ \\
\hline
\end{tabular}




\begin{tabular}{|c|c|c|c|c|}
\hline 146.90 & $\mathrm{~A}_{\mathrm{g}}$ & 8.75 & $\begin{array}{l}\text { Complex torsion (primarily } \\
\text { ether/alkyl chain) }\end{array}$ & bac \\
\hline 150.13 & $\mathrm{~B}_{\mathrm{g}}$ & 93.16 & $\begin{array}{l}\text { Complex torsion (primarily } \\
\text { ether/alkyl chain) }\end{array}$ & bac \\
\hline 150.61 & $\overline{A_{g}}$ & 109.56 & Carbazole bending/torsion & $\mathrm{ca}$ \\
\hline 153.86 & $\mathrm{~B}_{\mathrm{g}}$ & 73.47 & Carbazole torsion & $\mathrm{a}$ \\
\hline 160.80 & $\mathrm{~A}_{\mathrm{g}}$ & 64.05 & Complex torsion & $\mathrm{a}$ \\
\hline 177.56 & $\mathrm{~A}_{\mathrm{g}}$ & 50.61 & $\begin{array}{l}\text { Complex torsion (primarily } \\
\text { ether/alkyl chain) }\end{array}$ & $\mathrm{ac}$ \\
\hline 178.68 & $\mathrm{~B}_{\mathrm{g}}$ & 0.65 & $\begin{array}{l}\text { Complex torsion (primarily } \\
\text { ether/alkyl chain) }\end{array}$ & ba \\
\hline 183.87 & $\mathrm{~B}_{\mathrm{g}}$ & 15.93 & $\begin{array}{l}\text { Complex torsion (primarily } \\
\text { ether/alkyl chain) }\end{array}$ & bc \\
\hline 191.16 & $\mathrm{~A}_{\mathrm{g}}$ & 82.3 & $\begin{array}{l}\text { Complex torsion (primarily } \\
\text { ether/alkyl chain) }\end{array}$ & bca \\
\hline 195.38 & $\mathrm{~A}_{\mathrm{g}}$ & 18.68 & $\begin{array}{l}\text { Complex torsion (primarily } \\
\text { ether/alkyl chain) }\end{array}$ & bac \\
\hline 198.23 & $\mathrm{~B}_{\mathrm{g}}$ & 0.56 & $\begin{array}{l}\text { Complex torsion (primarily } \\
\text { ether/alkyl chain) }\end{array}$ & ba \\
\hline 208.92 & $\mathrm{~A}_{\mathrm{g}}$ & 24.51 & Complex torsion & ba \\
\hline 208.96 & $\mathrm{~B}_{\mathrm{g}}$ & 3.74 & Complex torsion & $\mathrm{bc}$ \\
\hline 231.05 & $A_{g}$ & 13.45 & $\begin{array}{l}\text { Complex torsion (primarily amino } \\
\text { group in the ether/alkyl chain) }\end{array}$ & bc \\
\hline 233.39 & $\mathrm{~B}_{\mathrm{g}}$ & 14.46 & $\begin{array}{l}\text { Complex torsion (primarily amino } \\
\text { group in the ether/alkyl chain) }\end{array}$ & bac \\
\hline 240.04 & $\mathrm{~B}_{\mathrm{g}}$ & 26.38 & $\begin{array}{l}\text { Complex torsion (primarily } \\
\text { ether/alkyl chain) }\end{array}$ & $\mathrm{bc}$ \\
\hline 242.42 & $\mathrm{~A}_{\mathrm{g}}$ & 14.28 & Complex torsion & $\mathrm{ac}$ \\
\hline 249.67 & $\mathrm{~B}_{\mathrm{g}}$ & 9.78 & $\begin{array}{l}\text { Complex torsion (primarily amino } \\
\text { group in the ether/alkyl chain) }\end{array}$ & cba \\
\hline 250.34 & $\mathrm{~A}_{\mathrm{g}}$ & 10.89 & $\begin{array}{l}\text { Complex torsion (primarily amino } \\
\text { group in the ether/alkyl chain) }\end{array}$ & bca \\
\hline 260.94 & $A_{g}$ & 2.63 & $\begin{array}{l}\text { Complex torsion (primarily } \\
\text { methoxy group) }\end{array}$ & bc \\
\hline 261.56 & $\mathrm{~B}_{\mathrm{g}}$ & 1.5 & $\begin{array}{l}\text { Complex torsion (primarily } \\
\text { methoxy group) }\end{array}$ & $\mathrm{b}$ \\
\hline 274.35 & $A_{g}$ & 5.62 & $\begin{array}{l}\text { Complex torsion (primarily } \\
\text { ether/alkyl chain) }\end{array}$ & ba \\
\hline 274.96 & $\mathrm{~B}_{\mathrm{g}}$ & 16.75 & $\begin{array}{l}\text { Complex torsion (primarily } \\
\text { ether/alkyl chain) }\end{array}$ & bc \\
\hline
\end{tabular}



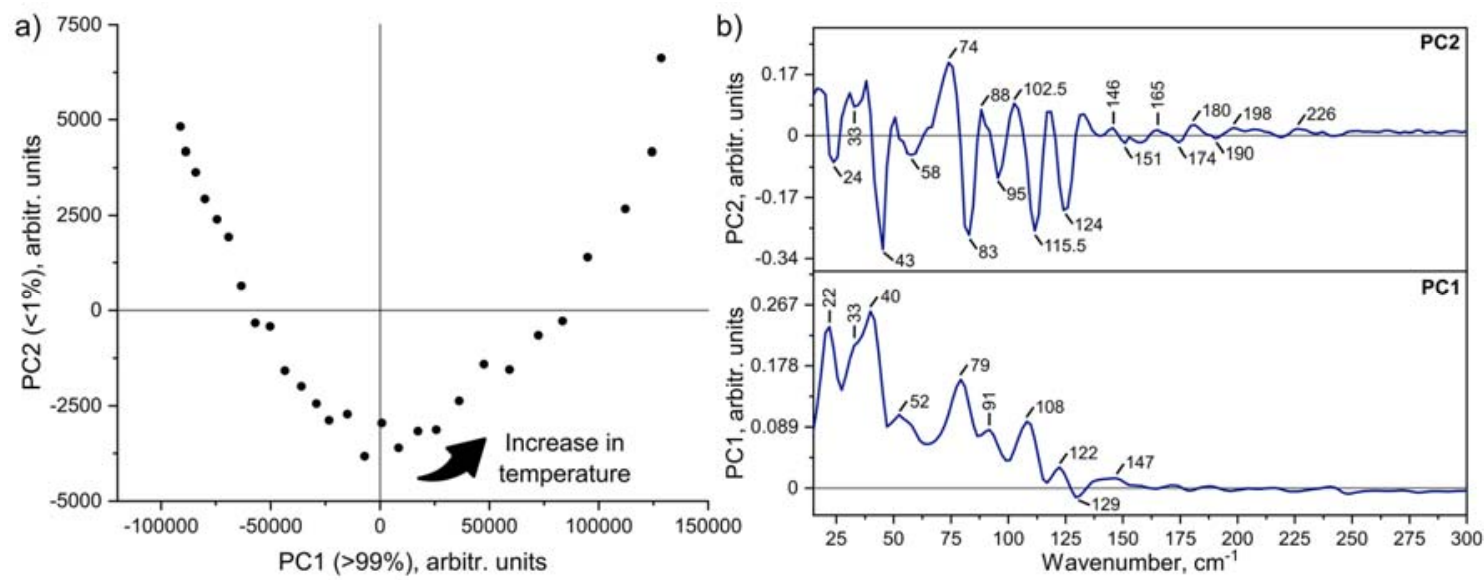

Figure S22. (a) Scores plot of PC1 versus PC2 and (b) the first two loadings plots from principal component analysis (PCA) of the variable temperature low-frequency Raman spectra of carvedilol (form II).

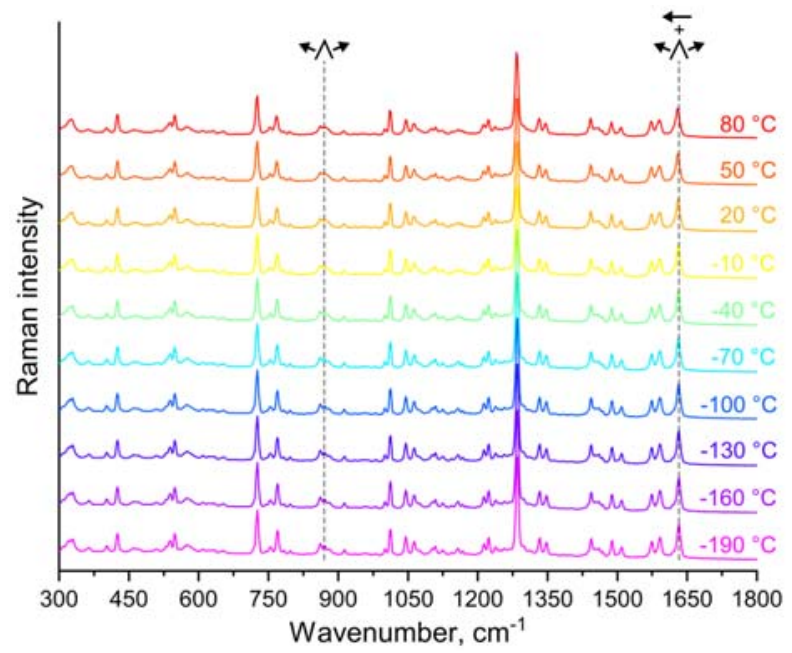

Figure S23. Mid-frequency Raman spectra of carvedilol (form II) in a broad temperature range.
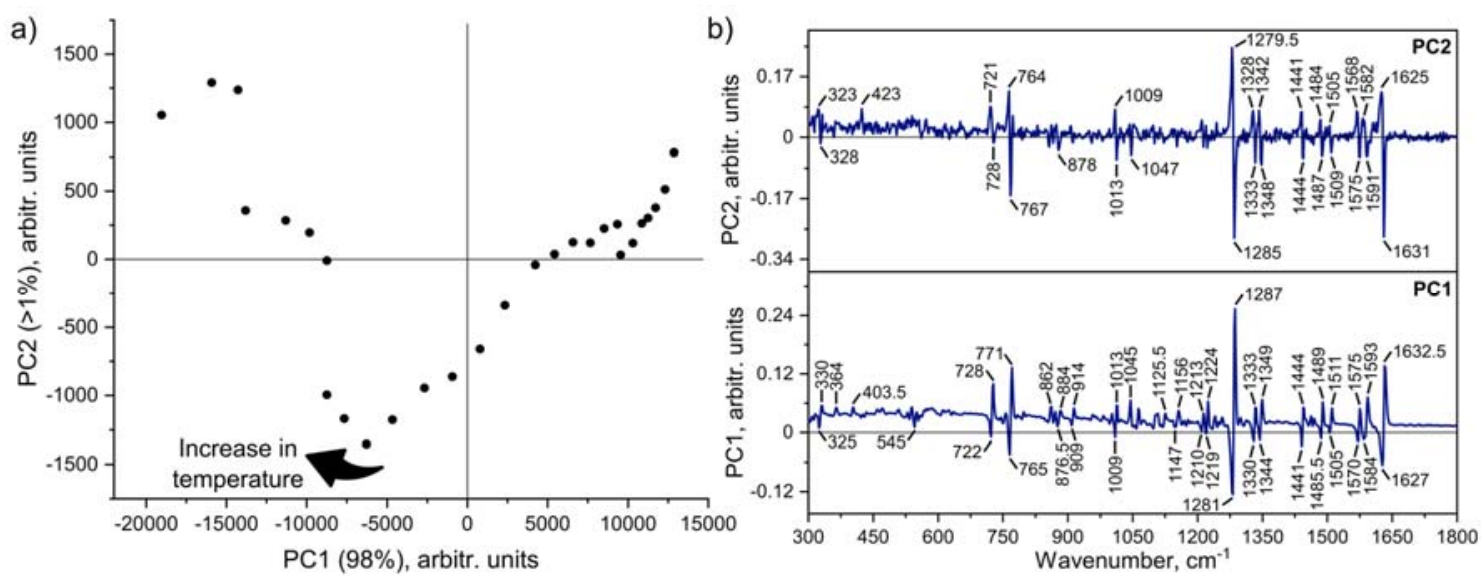

Figure S24. (a) Scores plot of PC1 versus PC2 and (b) the first two loadings plots from principal component analysis (PCA) of the variable temperature mid-frequency Raman spectra of carvedilol (form II). 

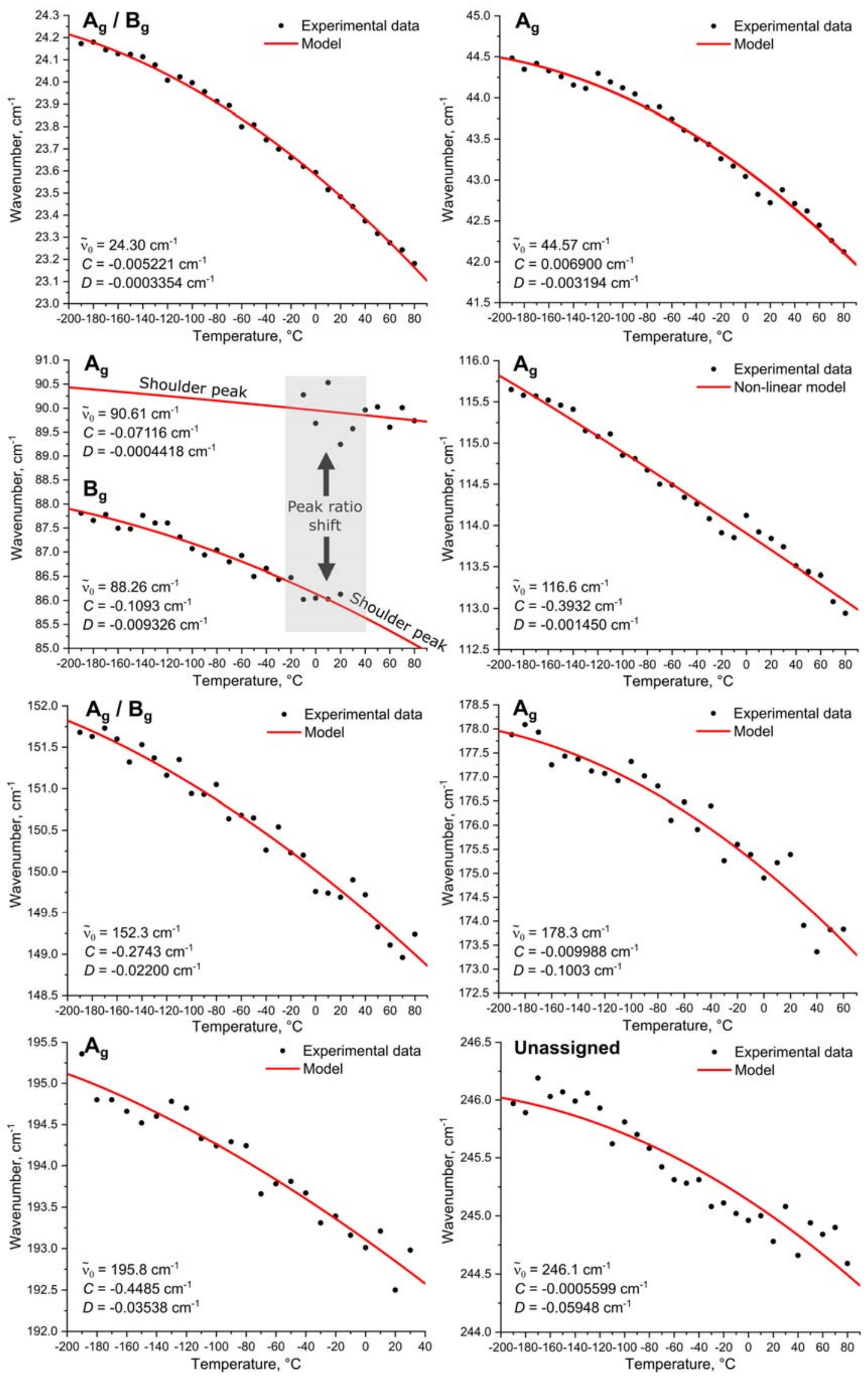
Figure S25. The modelling of temperature dependence of individual LFR peaks of carvedilol (form II) as compared to the experimental results. 